\title{
Metanálisis sobre la eficacia de los tratamientos antihipertensivos
}

\author{
Metanalisis about the efficacy of antihipertesive therapies
}

Law M y col. BMJ 2009;338:b1665

\section{Objetivo}

Determinar la eficacia de diferente clases de drogas antihipertensivas para prevenir enfermedad coronaria (EC) y accidente cerebrovascular (ACV) y los mejores candidatos a recibir tratamiento.

\section{Fuentes de Datos}

Medline (1966 a diciembre 2007) y Base de datos de la Colaboración Cochrane hasta la misma fecha.

\section{Selección de Estudios}

Ensayos clínicos aleatorizados que hubieran evaluado drogas antihipertensivas y registrado incidencia de de EC y ACV. Fueron excluidos estudios que hubieran incluido pacientes con insuficiencia renal crónica o con menos de cinco eventos registrados.

\section{Extracción de Datos}

Se registró el número de participantes que tuvieron eventos coronarios (infarto agudo de miocardio o muerte súbita) y cerebrovasculares (hemorrágicos o isquémicos).

Se determinó la diferencia en el promedio de tensión arterial (TA) de los pacientes de cada grupo de cada ensayo clínico a través de la resta de dichos valores antes y después de la intervención evaluada. Dos autores independientes analizaron los datos y el análisis se hizo por intención de tratar.

\section{Resultados Principales}

Fueron analizados 147 estudios y los riesgos relativos (RR) fueron estandarizados para una reducción de tensión arterial sistólica (TAS) de $10 \mathrm{mmHg}$ y una diastólica (TAD) de $5 \mathrm{mmHg}$. Los principales resultados se describen en las tablas 1 y 2 , y en la figura 1.

Tabla 1: efecto de los beta-bloqueantes para la prevención de nuevos eventos cardiovasculares de acuerdo al tiempo del inicio del tratamiento con los mismos y a la presencia o no de antecedentes coronarios en la población estudiada.

\begin{tabular}{|c|c|c|c|c|c|}
\hline \multirow{2}{*}{$\begin{array}{l}\text { Inclusión de } \\
\text { beta-bloqueantes }\end{array}$} & \multirow{2}{*}{\multicolumn{2}{|c|}{$\begin{array}{l}\text { De acuerdo al antecedente de enfermedad coronaria (EC) y/o infarto } \\
\text { agudo de miocardio (IAM) y al momento del inicio del tratamiento }\end{array}$}} & \multicolumn{2}{|c|}{ Número } & \multirow[t]{2}{*}{ RR (IC95\%) } \\
\hline & & & Estudios & Eventos & \\
\hline \multirow[t]{4}{*}{$\mathrm{Si}$} & \multirow{3}{*}{$\begin{array}{c}\text { Con antecedentes } \\
\text { coronarios }\end{array}$} & Sin discriminar por grupo & 37 & 2524 & $0,71(0,66$ a 0,78$)$ \\
\hline & & Tratamiento precoz luego de un IAM & 27 & 2155 & $0,69(0,63$ a 0,76$)$ \\
\hline & & Tratamiento tardío luego de un IAM & 11 & 369 & $0,87(0,71$ a 1,06$)$ \\
\hline & \multicolumn{2}{|l|}{ Sin antecedente coronarios } & 6 & 851 & $0,89(0,78$ a 1,02$)$ \\
\hline \multirow[t]{2}{*}{ No } & \multicolumn{2}{|c|}{ Con antecedentes coronarios } & 37 & 5834 & $0,85(0,79$ a 0,91$)$ \\
\hline & \multicolumn{2}{|c|}{ Sin antecedentes coronarios } & 24 & 3217 & $0,85(0,81$ a 0,89$)$ \\
\hline
\end{tabular}

Tabla 2: efecto de la reducción de la tensión arterial en la prevención de enfermedad coronaria y cerebrovascular en pacientes con y sin antecedentes cardiovasculares (prevención primaria y secundaria).

\begin{tabular}{|c|c|c|c|}
\hline \multirow{2}{*}{$\begin{array}{l}\text { Antecedentes de la } \\
\text { población incluida }\end{array}$} & \multirow[t]{2}{*}{ Número de estudios } & \multicolumn{2}{|c|}{$\begin{array}{c}\text { Disminución del riesgo (RR e IC95\%) de eventos por unidad de } \\
\text { reducción estandarizada de TA }\end{array}$} \\
\hline & & Coronarios & Cerebrovasculares \\
\hline Sin enfermedad cardiovascular & 27 & $0,79(0,72$ a 0,86$)$ & $0,54(0,45$ a 0,65$)$ \\
\hline Enfermedad coronaria & 37 & $0,76(0,68$ a 0,86$)$ & $0,65(0,53$ a 0,80$)$ \\
\hline Enfermedad cerebrovascular & 13 & $0,79(0,62$ a 1,00$)$ & $0,66(0,56$ a 0,79$)$ \\
\hline Resultado global & 72 & $0,78(0,73$ a 0,83$)$ & $0,59(0,52$ a 0,67$)$ \\
\hline
\end{tabular}

a10 mmHg para la presión sistólica y $5 \mathrm{mmHg}$ para la diastólica.

Cuando fueron comparados los resultados de cada grupo de drogas contra las demás con el objetivo de evaluar si existía algún efecto pleiotrópico preventivo de alguna droga, más allá del resultante de la disminución de la tensión arterial, sólo se observó que el tratamiento con betabloqueantes se asoció a un riesgo levemente superior de accidente cerebrovascular (RR 1,18 ; IC95\% 0,82 a 1,09). Por otro lado, el tratamiento con bloqueantes cálcicos se asoció a un riesgo levemente menor para el mismo resultado (RR 0,91; IC95\% 0,84 a 0,98); sin diferencias significativas para el resto de las comparaciones. 
Figura 1: reducción de incidencia de enfermedad cardiovascular en relación a la reducción de la presión arterial sistólica según la dosis y combinación de drogas, la presión sistólica previa al tratamiento y la edad.

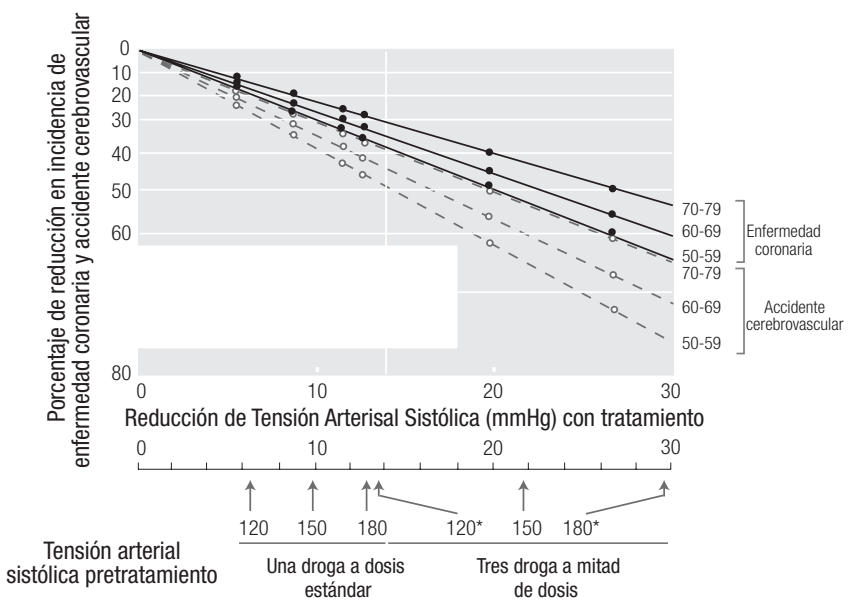

\section{Conclusiones}

Todas las clases de antihipertensivos demostraron reducir en forma similar el riesgo de enfermedad cardiovascular, confirmándose que los beta-bloqueantes tienen un especial efecto protector en pacientes con antecedentes de enfermedad coronaria, pero limitado a los primeros dos años posteriores al evento.

Disminuir los niveles de tensión arterial es beneficioso sin importar los valores previos al tratamiento, demostrándose que el riesgo de eventos disminuye, incluso en pacientes que tenían niveles de tensión arterial previos al tratamiento dentro del "rango de la normalidad".

El efecto antihipertensivo de las drogas explica por sí solo el efecto preventivo de las mismas; y que el uso de tres drogas a la mitad de su dosis máxima es más efectivo que el utilizar sólo una a una dosis estándar.

Palabras claves: drogas antihipertensivas, enfermedad coronaria, accidente cerebrovascular.

Key words: anti hypertensive agents, coronary heart disease, stroke.

Key words: anti hypertensive agents, coron

Conflicto de intereses: los autores tienen la patente de la píldora combinada para reducir riesgo cardiovascular.

\section{Comentario}

Dado que todos los grupos de drogas antihipertensivas evidenciaron el mismo efecto preventivo, sería razonable elegir el fármaco de acuerdo a sus contraindicaciones, efectos adversos y costos y teniendo en cuenta, según las características de cada paciente.

Dado que en prácticamente toda la población tratada pudo documentarse algún efecto beneficioso, los autores plantean que el uso de drogas antihipertensivas no se debería limitar a pacientes hipertensos. Esto supone cuestionamientos al paradigma actual de tratamiento de la hipertensión. Por ejemplo, podríamos vernos tentados a concluir que dejaría de ser útil tomar la presión a nuestros pacientes durante su control periódico de salud con el objetivo de encontrar individuos hipertensos o de monitorear si alcanzaron los objetivos terapéuticos.
Por otro lado, los autores proponen tratar inicialmente a los pacientes con tres drogas a la mitad de dosis usual, en vez de comenzar con una droga e ir adicionando otras, hasta lograr valores adecuados de tensión arterial.

\section{Conclusiones de la comentadora}

Los resultados de este metanálisis concuerdan con la estrategia poblacional de usar una "polipíldora"1 con tres drogas antihipertensivas a la mitad de su dosis usual, aspirina y estatina, para la prevención primaria de enfermedad cardiovascular; cuyo mayor efecto adverso sería la medicalización de pacientes sanos.

Ver glosario*

Tamara Sigal [ Servicio de Medicina Familiar y Comunitaria del Hospital Italiano de Buenos Aires. tamara.sigal@hospitalitaliano.org.ar ]

Sigal T. Metanálisis sobre el uso de drogas antihipertensivas para la prevención de enfermedad cardiovascular. Evid Act Pract Ambul. $13(2)$ 50-51. Abr-Jun 2010. Comentado de: Law M y col. Use of blood pressure lowering drugs in the prevention of cardiovascular disease: meta-analysis of 147 randomised trials in the context of expectations from prospective epidemiological studies. BMJ 2009;338:b1665. PMID 19454737

Bibliografía

1.Dreksler C. Vicente Lago I. El uso de una polípíldora podría mejorar la adherencia al tratamiento de los factores de riesgo cardiovascular. Evid Act Pract Ambul. 12(3) 8687. Jul-Sep 2009. Comentado de: Yusuf S y col. Effects of a polypill (polycap) on risk factors in middle-aged individuals without cardiovascular disease (TIPS): a phase II, double-blind, randomised trial. Lancet 2009 Apr 18; 373, 1341-1351. PMID: 19339045 Disponible en URL: http://www.thelancet.com/journals/lancet/article/PIIS01406736(09)60611-5/fulltext 